\title{
Pilot evaluation of the therapeutic effect of Silver Diamine Fluoride (SDF) in arresting dental caries in the primary teeth of Cambodian slum children
}

\author{
Mengkheng Chray, Shunhour Khorn, Sreykhouch Da, Bathsheba Turton*, \\ Callum Durward \\ Department of Dentistry, Faculty of Health Sciences, University of Puthisastra, \#55 Street 180, \\ Phnom Penh, Cambodia. \\ *Email: bethy.turton@gmail.com
}

\begin{abstract}
Silver Diamine Fluoride (SDF) is a clinically applied treatment that controls active dental caries and aids in preventing further progression of the caries process. Aim of study. To establish the effectiveness of SDF in arresting primary tooth caries in 6-12-year-old Cambodian children. A longitudinal observational study was conducted using a convenience sample of 167 children from Api Wat Meanchey primary school who were recruited following consent. Participants received two applications of SDF (Cariestop 30\%, Biodinamica, Ibipora, Brazil) at six-month intervals and follow-up examinations after 1 year. Oral health education was provided to the participants at the beginning of the study and a school tooth brushing program was in place. $118(70.7 \%)$ of the participants were followed up after one year of which $56.8 \%$ were female. The mean $\mathrm{dmft} / \mathrm{DMFT}$ at baseline was 10.5 (SD 7.6) / 4.6 (SD 4.9). At follow up $82.5 \%$ of open carious lesions were arrested. Application of $30 \%$ SDF solution two times per year was an effective way to manage caries in this very high caries risk population.
\end{abstract}

Keywords: arrest of caries, dental caries, cambodia, silver diamine fluoride, primary teeth

\section{Introduction}

According to the results of the first National Oral Health Survey in 1991, the mean DMFT of 12-year-old Cambodian children was 1.6. Very few carious teeth were restored. The situation was much worse in the 2011 National Oral Health Survey when the 12-year DMFT rose to 3.6, and for primary teeth at age 6 the mean dmft was 9.0 [1]. The survey noted that sugar consumption was generally high, and that tooth brushing was introduced late.

It is now well recognised internationally that oral health among schoolchildren should be a high priority, since it can affect their quality of life and general health and nutrition [2]. One of the most effective ways to address oral health problems in children is to target school groups and introduce school oral health programs which focus on caries prevention and management at population level. In Cambodia a number of initiatives have been implemented in recent years including $S E A L$ Cambodia [3] and Fit for School [4]. A more recent initiative known as 'Healthy 
Kids Cambodia' has been started which differs from the other two approaches in that it is more comprehensive and involves progressive delivery of dental therapeutic interventions including the use of Silver Diamine Fluoride (SDF) to arrest primary tooth caries.

One of the key features of caries experience in Cambodian children is that the vast majority of lesions go untreated, and this has now been shown to be associated with failure of children to thrive [4,5]. In addition, untreated dental caries in children has also been shown to be associated with reduced quality of life, intermittent pain and infection, space loss, inattention and absenteeism from school, social problems and problems with speech development [6].

Arrested dentine caries is clinically characterized by an increase in hardness and a dark brown or black coloration of the lesion. Clinical studies show that caries in primary teeth can be arrested to varying degrees by brushing with fluoride toothpaste [7], having fluoridated agents professionally applied (such as fluoride varnish (FV) and SDF), and sealing cavities with fluoride-releasing glass ionomer cement $[8,9]$. SDF has been shown to be more effective than FV at arresting caries in primary teeth9 and more effective (relative risk, 67\%) than Interim Restorative Treatment using a glass ionomer cement (relative risk, 39\%) [10].

Silver solutions have long been used in clinical dentistry for arresting cavitated lesions and also preventing new lesions [11]. Clinical studies have shown that SDF can be used to prevent and arrest coronal caries in both primary and permanent teeth as well as to manage or prevent tooth root caries for elderly patients [12]. SDF can contribute to inhibition of demineralization, and promotion of remineralization. In addition, there is evidence that the presence of silver inhibits collagenases that might otherwise break down dentine collagen in the caries process [9].

As well as arrest of caries, SDF can be used to prevent new lesions and also to reduce dentine hypersensitivity. The preventive effect has been documented in the pits and fissures of first permanent molars [11]. In addition to this, SDF has been effective at reducing dentine hypersensitivity in adults when applied 2-3 times per year [13].

There are differences in arrest rate depending on the concentration of SDF used and the frequency of application. The use of 30\% Cariestop SDF was shown to be more effective in arresting coronal caries in the primary teeth of children and in preventing root caries in elderly patients than the $12 \%$ concentration $[14,15]$. In addition, two applications of SDF were shown to be more effective than a single application, and higher plaque scores were associated with lower arrest rates $[8,16]$.

Advantages of using SDF include: it is easy to use; it does not require electricity or powered instruments; it is fast to apply; it is low cost; it does not require local anesthetic; and the literature has reported predictable results. In addition, SDF can also be used for children who have dental anxiety, for "fissure protection" on sound permanent molars, and for management of root lesions [17,18].

Although SDF is clinically effective, there are some draw backs. The most commonly reported adverse effect of SDF is black staining of carious dentine $[19,20]$. SDF stains carious dentine permanently black, particularly when higher concentrations or repeated applications of SDF are applied [19]. The dark staining is due to the formation of silver phosphate in the surface layers of dentine as well 
as the demineralized enamel. A pre-treatment discussion about the pros and cons of SDF treatment with the children and their parents is vital to ensure that they are satisfied with the therapy and will not be upset when the treated lesions turn a black color.

The other disadvantage of SDF is gum irritation. This is a mild but transient gingival irritation which can sometimes occur after SDF application [14,20]. If spilt onto the oral mucosa a white erosive lesion may form, however this will heal spontaneously within 2 days. Prevention of gingival irritation by applying Vaseline over the gingival margin has been proposed by several authors to prevent irritation, however others do not follow this practice $[10,18,19]$.

Measures should be taken to prevent accidental spillage of SDF onto the face, eyes, clothes or skin of the patient or operator. Contact with skin can produce a brown or black stain which is not permanent. Contact dermatitis (observed sometimes after sodium fluoride varnish application) has never been reported after application of SDF. A literature search found that there were no reported cases of acute toxicity or significant adverse effects after professional application of SDF. There is little risk of excess silver accumulation causing toxicity because only a minute amount of SDF is applied to the dental tissues either semi-annually or annually [20,21].

In addition to its topical effects, there has been some discussion as to whether SDF might illicit an unfavourable pulpal response following application, and whether there might be a risk of fluoride overdose. However, previous studies have indicated that SDF does not cause severe pulpal reaction, and no severe pulpal damage has been reported [20]. With regards to the risk of ingesting excessive fluoride, since only a very small amount of SDF solution is applied to a carious lesion, researchers have concluded that the systemic exposure to fluoride using this technique is well below the concentrations associated with toxicity [21].

SDF treatment can potentially increase access to care, improve oral health, and reduce the need for emergency care and treatment. In community settings the use the SDF can arrest most carious lesions at low cost, and avoid the need go to a dental clinic in many cases. This is especially important where children are from low income families or where conventional dental services are not readily available [22]. Although many studies have been conducted in other settings, there have been no studies to examine the effectiveness of SDF in the Cambodian setting, where children have extreme levels of dental caries experience.

\section{Methods}

A convenience sample of 167 children from Api Wat Meanchey primary school in Phnom Penh were recruited into the Healthy Kids Cambodia program and followed for one-year following consent. Participants received two applications of SDF Cariestop 30\%, Biodinamica, Ibipora, Brazil at a six month interval across the course of 1-year as part of the Healthy Kids Cambodia Project. Api Wat Meanchey is a slum community that is supported by a Non-governmental organisation (NGO) called 'Matesabroad'. That NGO works together with another called One-2-One Cambodia in implementing their health approach.

The Healthy Kids Cambodia provides dental interventions in a graduated way with handwashing, tooth brushing and SDF first, Atraumatic restorations and sealants second, then conventional dentistry third. The present paper focuses on the 
efficacy of the SDF therapy only. Ethical approval was provided by the Cambodian National Ethics Committee for Health Research. Participants were included if they attended the school. Exclusion was based on lack of consent, lack of cooperation, inability to isolate the teeth with cotton rolls, attendance at another school, or an age higher than 16-years.

\subsection{Clinical procedure}

SDF was placed by volunteer dentists, dental students, and dental nurses (therapists) who were members of teams working in the Healthy Kids Cambodia project. Participants received two applications of SDF (Cariestop 30\%) at six-month intervals across the course of one year. SDF was placed on any cavited lesion in permanent and primary teeth except for those lesions on permanent anterior teeth, and lesions which had reached the pulp. The clinical procedure included the following steps: (1) Place cotton roll isolation, (2) Clean and dry the carious lesions (one quadrant at a time) using wet/dry cotton pellets (no caries removal), (3) Place SDF by gently agitating the wet microbrush in the target areas, (4) Maintain isolation and wait for one minute, (5) Advise the patient to avoid eating or drinking for 1 hour.

\subsection{Baseline and follow-up examinations}

Three examiners were calibrated at baseline and again at the 1-year follow-up, rendering Kappa scores above 0.85 . Examinations were conducted with the child in the supine position using a head-light to enhance visibility. Teeth were scored for the presence of a cavitated carious lesion, restoration, or missing due to caries. The PUFA index was used to record the consequences of untreated caries [23]. A carious lesion was said to be "arrested" if a blunt periodontal probe did not penetrate the lesion with moderate pressure or if the colour was black.

\subsection{Data management}

Data were first collected on paper forms and then entered into SPSS (IBM version 22.0) for analysis. Descriptive analysis was carried out followed by comparison of lesion progression by group membership. The Chi-squared test was used to examine differences in proportions and the Kruskal-Wallis test was used to examine differences in means.

\section{Results}

Table 1 presents data on the sociodemographic characteristics of patients. One-third of children were in the 8- to 9-year-old age-group. There was no significant difference in participation by gender. 118 out of 167 children were followed up $(70.7 \%)$. 
Table 1. Socio demographic characteristics of participants ${ }^{a}$

\begin{tabular}{|c|l|l|l|}
\hline \multicolumn{2}{|c|}{ Male } & \multicolumn{1}{c|}{ Female } & \multicolumn{1}{|c|}{ Total $^{\text {b }}$} \\
\hline Age-group & \multicolumn{3}{|c|}{} \\
\hline Under 6-years & $1(20.0)$ & $4(80.0)$ & $5(4.2)$ \\
\hline 6-to 7-years & $12(57.1)$ & $9(42.9)$ & $21(17.8)$ \\
\hline 8-to 9-years & $12(34) 1$. & $27(65.9)$ & $41(34.7)$ \\
\hline 10-to 11-years & $8(34.8)$ & $15(65.2)$ & $23(19.5)$ \\
\hline 12-to 16-year & $16(57.1)$ & $12(42.9)$ & $28(23.7)$ \\
\hline Total & $51(43.2)$ & $67(56.8)$ & $118(100.0)$ \\
\hline
\end{tabular}

${ }^{\mathrm{a} B r a c k e t s ~ c o n t a i n}$ row percentages unless otherwise indicated

${ }^{\mathrm{b}}$ Brackets contain column percentages

Table 2 presents data on the clinical characteristics of participant at baseline. There were no significant differences by gender at baseline. There was a significant difference in caries experience by age-group.

Table 2. Clinical Characteristics of participants at follow-up

\begin{tabular}{|c|c|c|c|}
\hline & $\begin{array}{c}\text { Any Caries } \\
\mathbf{N}(\%)\end{array}$ & $\begin{array}{c}\mathrm{dmft} \\
\text { mean }(\mathrm{SD})\end{array}$ & $\begin{array}{c}\text { DMFT } \\
\text { Mean (SD) }\end{array}$ \\
\hline \multicolumn{4}{|l|}{ Gender } \\
\hline Male & $49(96.1)$ & $6.7(5.3)$ & $2.4(2.9)$ \\
\hline Female & $66(98.5)$ & $6.4(5.4)$ & $2.3(2.0)$ \\
\hline \multicolumn{4}{|l|}{ Age-group $^{a}$} \\
\hline Under 6-years & $5(100.0)$ & $8.8(3.7)$ & $1.3(1.3)$ \\
\hline 6-to 7-years & $21(100.0)$ & $11.0(4.4)$ & $1.3(1.3)$ \\
\hline 8-to 9-years & $41(100.0)$ & $8.2(4.8)$ & $1.4(1.5)$ \\
\hline 10-to 11-years & $23(100.0)$ & $5.0(4.5)$ & $2.6(2.4)$ \\
\hline 12 years or older & $25(89.3)$ & $1.5(2.8)$ & $3.4(3.5)$ \\
\hline Overall & $115(97.5)$ & $6.5(5.3)$ & $2.3(2.4)$ \\
\hline
\end{tabular}

Table 3 presents arrest of caries by socio-demographic characteristics of participants. There were no differences in arrest rates by gender or age group. Four out of five lesions were successfully arrested.

Table 3. Arrest of caries by sociodemographic of participants at follow-up.

\begin{tabular}{|c|c|c|c|}
\hline & $\begin{array}{c}\text { Number of open le- } \\
\text { sions } \\
\text { Mean (SD) }\end{array}$ & $\begin{array}{c}\text { Number of arrested } \\
\text { lesions } \\
\text { (Mean SD) }\end{array}$ & $\begin{array}{c}\text { Proportion of ar- } \\
\text { rested lesions } \\
\% \text { (SE) }\end{array}$ \\
\hline Gender & $7.3(5.0)$ & $5.7(4.3)$ & $81.7(33.8)$ \\
\hline Male & $6.6(5.0)$ & $5.5(4.7)$ & $83.1(26.9)$ \\
\hline Female & $6.6(5.3)$ & $5.4(3.9)$ & $89.3(14.7)$ \\
\hline \multicolumn{4}{|c|}{ Age-group } \\
\hline Under 6-years & $10.5(4.6)$ & $9.1(4.7)$ & $82.9(25.9)$ \\
\hline 6-to 7-years & $8.1(4.9)$ & $6.7(4.9)$ & $83.0(30.5)$ \\
\hline 8-to 9-years & $4.9(2.8)$ & $4.5(2.6)$ & $71.6(36.7)$ \\
\hline 10-to 11-years & $4.1(4.6)$ & $2.5(2.6)$ & $82.5(30.0)$ \\
\hline 12 years or older & $6.9(4.9)$ & $5.6(4.5)$ & \\
\hline
\end{tabular}




\section{Discussion}

This longitudinal study indicates that SDF is an effective therapy for managing dental caries in a high-risk group of children in Cambodia. Before progression to further discussion of the findings of the paper, it is appropriate to consider the strengths and limitations of the present study. The major limitation of this study was the sampling. While there are many advantages to convenience sampling such as allowing investigators to quickly apply the interventions in one place to all the children, the risk is that the results could be biased and may not represent the wider population. The other issue with this investigation is that there was no control group, therefore we cannot be sure about the rate of arrest that would have occurred if participants did toothbrushing alone, or if there were no interventions at all. Thirdly, the operators were a diverse mix of dentists, dental students and dental nurses - all with varying amounts of training and skill. In saying that, there was a lot of supervision of students to ensure that the protocol was followed. Major strengths of the study were the high follow-up rate which increased the chance that we were able to observe the true effect of the intervention, and the very high consent rate.

\subsection{Caries experience among the study sample}

The range of ages of children in this study was broad; however, the caries experience was severe for all groups. Those in the 12-year and above age-group represented children who had cavitated lesions on an average of 3.4 teeth, mostly first permanent molars. The reason that these children had such a severe caries experience in their permanent dentition may be due to the environment in and around the school. In this community sellers make money by selling sweet snack foods to factory workers and school children. One can observe many sellers at the school gate and even on the school grounds selling non-nutritious foods and drinks to children during their break times. Those in the older age-groups are further at risk from a high sugar diet as most get pocket money on a regular basis and can select foods and beverages by themselves with less restriction. Another contributor to this very high rate of tooth decay could be that many of the children come from homes where there is no running water, which makes it difficult to routinely carry out oral hygiene practices at home. In addition, some families may not be able to afford to buy good quality fluoride toothpaste and toothbrushes for their children.

\subsection{Caries Management with SDF}

In this study, SDF (30\% Cariestop) applied 2 times per year by a dentist, dental nurse (therapist) or dental student rendered an $82.5 \%$ arrest rate. This rate is one of the highest that has been reported; arrest rates in other studies range from $40 \%-90 \%$ with bi-annual applications [15]. Part of the reason for the high success rate could be due to good adherence to the SDF protocol. The authors believe that one of the most important aspects of the protocol is the 1-minute waiting time following application of the SDF to the dried carious lesion. This waiting time allows for penetration of the liquid into the dentinal tubules and for the reaction between the silver ions and the surface of the tooth to be potentiated. Also, the SDF was allowed to stay on the tooth following application rather than being rinsed off (as has been 
recommended in some previous studies) resulting in prolonged exposure of silver diamine fluoride on the surface of the lesion [9].

\subsection{Is caries arrest an appropriate measure of success?}

In this study, arrest of caries was defined based on the hardness and colour of the lesion at follow-up. However, these variables do not quantify changes in the size of the lesion, and there is no way to know how long a lesion might stay 'arrested'. This study involved only a 1-year follow-up and perhaps it would be useful for multiple measurements to be made over a longer time period in order to more accurately describe the caries activity. Furthermore, although the colour of a lesion might be relatively easy to quantify, it is more difficult to calibrate examiners on the hardness of a lesion. This may not be an issue if the outcome is viewed from the caries balance perspective. It is likely that changes in the clinical characteristics of a lesion (colour and hardness) indicate a transition from a caries-active state, in which pathological factors are dominating, towards a caries-inactive state, in which protective factors can work together to slow the progression of disease. If the caries activity can be reduced, then the treatment has been successful.

\subsection{Was the SDF appropriate for this setting?}

There are two key arguments for the use of SDF in this setting. The first argument relates to the management of caries, and the second argument to the allocation of resources. Participants in this study had an extreme caries experience and conventional restorative techniques would have potentially failed if they had been attempted without implementing other caries management measures first. In addition to the SDF applications, the children participated in daily supervised tooth brushing and received GIC fissure sealants, as appropriate. It is certain that most of these children from slum families would never be able to afford conventional dental treatment in a private dental clinic, and so the interventions provided in the Healthy Kids Cambodia project are helping to meet their basic oral health needs in a very appropriate, low-cost and child-friendly way. In addition, the children have now become used to a school-based dental program focusing on Minimal Intervention Dentistry (MID) approach after multiple contacts with oral health professionals, and this has helped to reduce anxiety and has reinforced positive oral health behaviours.

The application of SDF was part of a wider school-based integrated health strategy known as 'Healthy Kids Cambodia'. This strategy involves cooperation between Non-Governmental Organisations lead by One-2-One Cambodia, and the University of Puthisastra, in partnership with the School Health Department at the Ministry of Youth Education and Sport (Cambodia). This strategy is divided into three levels: The first involves daily handwashing, tooth brushing and Arrest of Caries Treatment (ACT) with SDF; the second involves Atraumatic Restorative Technique (ART) restorations and GIC fissure sealants; and the third involves more comprehensive dentistry (including conventional restorations, root canal treatments and extractions). The first and second levels are provided in the school environment, while the third level of treatment can be provided to children using mobile equipment in the school, or at a fixed dental clinic. 
Another reason for using SDF in this setting concerns the affordability of SDF treatment. SDF treatment for a child involves the use of 2400 rhiel worth of materials (approximately USD 0.57) and in this study an average of approximately 6 lesions per child were successfully managed with two applications. Because most of the lesions could be effectively managed in this way, and since very few lesions were still active at the end of 1-year, the NGO supporting the school was able to allocate resources so that those with the most severe needs could have more comprehensive dentistry provided at a local university dental clinic.

\section{Conclusion}

This study involved children with a very high caries experience from one Cambodian school participating in the Healthy Kids Cambodia project. Results showed that application of 30\% SDF solution (Cariestop) two times per year was an effective way to manage caries in this population. The success is thought to be due to the good adherence to the SDF protocol and the concurrent implementation of other caries management interventions (e.g. daily brushing) included in the Healthy Kids Cambodia strategy. This study shows that SDF can be cost-effective and appropriate in a population with extreme caries experience in Cambodia.

\section{Acknowledgements}

The authors would like to acknowledge the volunteers who placed the SDF for the school children. Also, the non-governmental organisation (Mates Abroad) which funded the materials for the intervention, and One-2-One Cambodia which coordinated the intervention.

\section{References}

1. Chher T, Turton BJ, Hak S, Beltran E, Courtel F, Durward C, Hobdell M. Dental caries experience in Cambodia: Findings from the 2011 Cambodia National Oral Health Survey. J Int Oral Health. 2016;8(1):1.

2. Allukian Jr M. The neglected epidemic and the surgeon general's report: a call to action for better oral health Am J Public Health. 2008 Sep;98(9 Suppl):S82-5

3. Turton B, Durward C, Manton D. From 'Point and Pull' to 'Seal the Deal' - Transforming the way dental services are provided for disadvantaged children in Cambodia. Synopses 2017: in print.

4. Duijster D, Monse B, Dimaisip-Nabuab J, Djuharnoko P, Heinrich-Weltzien R, Hobdell M, Kromeyer-Hauschild K, Kunthearith Y, Mijares-Majini MC, Siegmund N, Soukhanouvong P. 'Fit for school'-a school-based water, sanitation and hygiene programme to improve child health: Results from a longitudinal study in Cambodia, Indonesia and Lao PDR. BMC Public Health. 2017;17(1):302.

5. Benzian H, Monse B, Heinrich-Weltzien R, Hobdell M, Mulder J, van Palenstein Helderman W. Untreated severe dental decay: a neglected determinant of low Body Mass Index in 12-year-old Filipino children. BMC Public Health. 2011 Dec;11(1):558.

6. Sheiham A. Dental caries affects body weight, growth and quality of life in pre-school children. Br Dent J. 2006;201(10):625.

7. Lo EC, Schwarz E, Wong MC. Arresting dentine caries in Chinese preschool children. Int J Ped Dent. 1998 Dec 1;8(4):253-60. 
8. Zhi QH, Lo EC, Lin HC. Randomized clinical trial on effectiveness of silver diamine fluoride and glass ionomer in arresting dentine caries in preschool children. J Dent. 2012;40(11):962-7.

9. Zhao IS, Gao SS, Hiraishi N, Burrow MF, Duangthip D, Mei ML, Lo EC, Chu CH. Mechanisms of silver diamine fluoride on arresting caries: a literature review. Int Dent J. 2017.

10. dos Santos Junior VE, de Vasconcelos FM, de Souza PR, Ribeiro AG, Rosenblatt A. Adverse events of the use of interim therapeutic in schoolchildren: silver diamine fluoride $\mathrm{x}$ interim therapeutic restorative-a pilot study. Revista Odonto Ciência.;27(1):2630 .

11. Liu BY, Lo EC, Chu CH, Lin HC. Randomized trial on fluorides and sealants for fissure caries prevention. Journal Dent Res. 2012;91(8):753-8.

12. Zhang W, McGrath C, Lo EC, Li JY. Silver diamine fluoride and education to prevent and arrest root caries among community-dwelling elders. Caries Res. 2013;47(4):284 90 .

13. Castillo JL, Rivera S, Aparicio T, Lazo R, Aw TC, Mancl LL, Milgrom P. The shortterm effects of diammine silver fluoride on tooth sensitivity: a randomized controlled trial. J Dent Res. 2011;90(2):203-8.

14. $\mathrm{Chu} \mathrm{CH}$, Lo EC, Lin HC. Effectiveness of silver diamine fluoride and sodium fluoride varnish in arresting dentin caries in Chinese pre-school children. J Dent Res. 2002;81(11):767-70.

15. Contreras V, Toro MJ, Elías-Boneta AR, Encarnación-Burgos MA. Effectiveness of silver diamine fluoride in caries prevention and arrest: a systematic literature review. General Dent. 2017;65(3):22.

16. Fung MH, Duangthip D, Wong MC, Lo EC, Chu CH. Arresting dentine caries with different concentration and periodicity of silver diamine fluoride. JDR Clin Translat Res. 2016;1(2):143-52.

17. Duangthip D, Chu $\mathrm{CH}$, Lo EC. A randomized clinical trial on arresting dentine caries in preschool children by topical fluorides - 18-month results. J Dent. 2016;44:57-63.

18. Horst JA, Ellenikiotis H, Milgrom PM, UCSF Silver Caries Arrest Committee. UCSF protocol for caries arrest using silver diamine fluoride: rationale, indications, and consent. J California Dent Assoc. 2016;44(1):16.

19. Yee R, Holmgren C, Mulder J, Lama D, Walker D, van Palenstein Helderman W. Efficacy of silver diamine fluoride for arresting caries treatment. J Dent Res. 2009;88(7):644-7.

20. Rosenblatt A, Stamford TC, Niederman R. Silver diamine fluoride: a caries "silver-fluoride bullet". . J Dent Res. 2009;88(2):116-25.

21. Mei ML, Chu CH, Lo EC, Samaranayake LP. Fluoride and silver concentrations of silver diamine fluoride solutions for dental use. Int J Paed Dent. 2013;23(4):279-85.

22. Chu CH, Lo EC. Promoting caries arrest in children with silver diamine fluoride: a review. Oral Health Preventive Dent. 2008;6(4).

23. Monse B, Heinrich - Weltzien R, Benzian H, Holmgren C, van Palenstein Helderman W. PUFA - an index of clinical consequences of untreated dental caries. Community Dent Oral Epidemiol. 2010;38(1):77-82. 